Meta

Journal des traducteurs

Translators' Journal

\title{
Variations autour de la francisation des désignations de fonction
}

\section{Julie Desgagné}

Volume 26, numéro 3, septembre 1981

URI : https://id.erudit.org/iderudit/002548ar

DOI : https://doi.org/10.7202/002548ar

Aller au sommaire du numéro

Éditeur(s)

Les Presses de l'Université de Montréal

ISSN

0026-0452 (imprimé)

1492-1421 (numérique)

Découvrir la revue

Citer cet article

Desgagné, J. (1981). Variations autour de la francisation des désignations de fonction. Meta, 26(3), 268-272. https://doi.org/10.7202/002548ar d'utilisation que vous pouvez consulter en ligne.

https://apropos.erudit.org/fr/usagers/politique-dutilisation/ 


\section{VARIATIONS AUTOUR DE LA FRANCISATION DES DÉSIGNATIONS DE FONCTION}

Si l'usage est roi,

les usagers ne le sont pas

$\mathrm{Au}$ Québec, où l'anglais et le français sont en contact, donc en situation d'interférence, la francisation des désignations de fonction soulève la question du rendu de la réalité en langue originale, c'est-à-dire en un français qui n'est pas une langue de traduction.

En effet, le traducteur dans sa recherche d'équivalents a, plus souvent qu'à son tour, rencontré des faux-amis sur sa route. C'est particulièrement le cas de nombreux mots anglais et français. Ces deux langues ayant un passé commun, le passage de l'une à l'autre est semé d'embûches.

La langue québécoise, singulièrement la langue des structures d'entreprise, porte encore, comme autant de cicatrices, les marques des pièges dans lesquels sont tombés nos premiers traducteurs, démunis qu'ils étaient de documentation en langue originale.

Examinons une structure d'entreprise américaine pour voir comment y est découpée la réalité «hiérarchique», en recourant, pour ce faire, aux désignations en langue originale.

Au sommet de la hiérarchie, se trouve le «board of directors» qui est «the governing body of a corporation». Un de ses membres, le «chairman (of the board)" en préside les réunions. À ce titre, il délègue son autorité à un «vicechairman". Du "chairman" on dit: "In actual practice, he usually is the most powerful member of the policy making decision group". On dit encore: "Frequently, he will also be the chief operating officer of the firm».

Lorsque tel n'est pas le cas, le «board of directors» délègue son autorité à un «president» qui est «the chief operating officer of the firm». 
Ouvrons ici une parenthèse. Le «chairman of the board» ou le «president» peuvent être «chief executive», c'est-à-dire «the top officer of a corporation»... "accountable to the board of directors for the activities and profits of the firm". Les définitions de "chief executive" précisent: "This job is commonly used to describe the responsibility which the job holder discharges rather than as a job title». Comme on le voit, cette appellation peut s'impliciter lorsque l'intéressé est déjà président-directeur général. Fermons la parenthèse.

Le «president», en tant que responsable de l'exploitation, délègue à son tour son autorité à des «vice-presidents" qui sont les «president's deputies in charge of particular locations or functions».

Ces «vice-presidents» commettent à leur tour leur autorité à des «department heads», «managers» ou «directors", "whose work is the management of a particular phase or activity within the business". Nous reviendrons sur le mot «director», car il mérite un traitement particulier.

Ces «managers» délèguent leur autorité à des «deputy managers» ou à des "supervisors" "who are first line managers who direct the activities of operative level employees».

Nous avons donc:

board of directors

chairman (of the board)

vice-chairman

president

vice-presidents

department heads; managers ; «directors"

deputy managers, «managers»

supervisors

senior employees

Examinons maintenant le découpage de la réalité «hiérarchique» dans une structure d'entreprise française en utilisant encore les désignations de fonction en langue originale, le français.

Au sommet, se trouve le conseil d'administration qui est «l'organe de direction des sociétés anonymes». Ce conseil est présidé par l'un de ses membres, le président du conseil d'administration qui, à ce titre, délègue son autorité à un vice-président.

Le président du conseil exerce, le plus souvent, la fonction de directeur général d'où l'appellation président-directeur général et, toutes les langues tendant à l'économie, l'appellation pure et simple de président. «En tant que directeur général, il est l'agent d'exécution des délibérations du conseil», donc responsable de l'exploitation «qui est la face économique de la gestion». Bref, il est celui qui fait marcher l'entreprise. "Il dispose des pouvoirs des plus étendus pour agir et représenter la société dans le cadre défini par l'objet social».

Il délègue son autorité à des directeurs qui sont des «collaborateurs immédiats de la direction générale en tant qu'ils participent à l'élaboration de la politique de l'entreprise en ce qui concerne leur domaine». Ils exercent leur autorité sur des organismes complexes appelés directions correspondant, en principe, à chaque fonction, ou à une implantation, de l'entreprise. «Eux-mêmes sont membres de la direction de l'entreprise». 
Les directeurs délèguent leur autorité à des chefs d'organisme: divisions ou services. Ces chefs exercent leur autorité dans un domaine opérationnel.

Ils délèguent leur autorité à des sous-chefs (ou chefs de subdivision).

Ces sous-chefs commettent leur autorité à des chefs de section ou à des employés principaux qui encadrent directement les exécutants.

Nous avons cette fois:

conseil d'administration

président (du conseil d'administration)

vice-président

directeur général

directeurs

chefs d'organismes: divisions ou services

subdivisions

sections

sous-sections

employés principaux

À première vue, force est de constater le découpage identique des deux réalités hiérarchiques; ensuite, la rigueur et la clarté des dénominations hiérarchiques françaises. Elles délimitent le domaine de la direction: direction générale, direction, et le domaine opérationnel. Ce faisant, elles réservent, de façon transparente, la désignation directeur aux personnes responsables de la direction de l'entreprise: le directeur général et ses collaborateurs : les directeurs fonction$n e l s$, c'est-à-dire chargés d'une fonction (ou d'une implantation).

Ajoutons toutefois que certains subordonnés de la direction générale ont qualité de directeurs hiérarchiques lorsqu'ils sont responsables DEVANT ELLE de l'exécution d'un programme, par exemple, la restructuration de l'entreprise. Elles précisent l'importance hiérarchique des organismes les uns par rapport aux autres: direction générale, direction, division, subdivision, section, sous-section. Et qui plus est, cette importance hiérarchique nettement définie caractérise le rang hiérarchique de ceux qui les commandent :

direction générale

directions

divisions

subdivisions

sections

sous-sections

directeur général

directeurs

chefs de division

chefs de subdivision

chefs de section

chefs de sous-section avons:

Si nous rapprochons ces désignations des désignations américaines, nous

directors

board of directors

chairman (of the board)

vice-chairman

president

vice-presidents

administrateurs

conseil d'administration

président (du conseil d'administration)

vice-président

department heads

directeur général

directeurs

managers or «directors »

chefs de division; chefs de service

deputy managers or «managers» sous-chefs; chefs de subdivision 
supervisors senior employees chefs de section employés principaux

Augmentons ce tableau comparatif des désignations en langue originale des désignations en langue de traduction qu'on observe au Québec. Nous avons:

$\begin{array}{ll}\text { ADMINISTRATION } & \begin{array}{l}\text { Langue originale } \\ \text { directors } \\ \text { board of directors } \\ \text { chairman of the board } \\ \text { vice-chairman }\end{array} \\ \text { DOMAINE } & \left.\begin{array}{l}\text { president } \\ \text { vice-presidents } \\ \text { OPERATIONNEL } \\ \text { department heads } \\ \text { «anagers } \\ \text { deputy managers } \\ \text { «managers» } \\ \text { supervisors } \\ \text { senior employees }\end{array}\right]\end{array}$

Langue originale
administrateurs
conseil d'administration
président (du conseil
d'administration)
vice-président
directeur général
directeurs
chefs de division;
chefs de service
chefs de subdivision;
sous-chefs
chefs de section
employés principaux

Langue de traduction administrateurs conseil d'administration président du conseil d'administration vice-président président vice-présidents chefs de service directeurs directeurs adjoints

superviseurs employés principaux employés seniors

Nous constatons dans le passage d'une langue à l'autre, plusieurs cas d'interférence, sans compter la perte de la rigueur qu'a la deuxième série de désignations en langue originale.

Examinons la colonne de gauche. Nous y constatons la présence de «directors» au sens de "department heads or managers». Nous avons consulté une vingtaine de dictionnaires de langue et de dictionnaires spécialisés, américains, britanniques et canadiens. Nous avons relevé ce terme dans un seul, un dictionnaire de la langue anglaise au Canada, qui définit ce mot ainsi : "a manager». On peut penser qu'il s'agit là d'un emprunt à la langue de traduction québécoise qui, nous l'avons vu, l'utilise improprement.

Observons maintenant la colonne du centre. Nous y relevons division qui entre en concurrence avec l'anglais "division». Mettons-les en équivalence: division signifie en anglais "department", "division" se rend en français par département. (Le département est la partie d'une organisation chargée de la gestion et ayant la responsabilité d'une activité globale spécifique, d'un domaine fonctionnel, d'un secteur géographique.)

Passons à la colonne de droite. Nous y relevons président au sens de directeur général et vice-président au sens de directeur. Nous y constatons la présence de directeur, donc de directeur adjoint, à un niveau inférieur à celui de la direction de l'entreprise; l'emprunt à l'anglais «senior» au sens de principal et l'emploi de superviseur dans un sens qu'il n'a pas. (Le superviseur est la personne qui contrôle un travail sans entrer dans le détail.)

La francisation des désignations de fonction est exemplaire en ce sens qu'elle illustre les dimensions linguistique et psycho-sociale de la francisation des entreprises.

Elle nous fait prendre conscience des traquenards qui hérissent le passage de l'anglais au français. En soi, cela devrait suffire à éveiller la vigilance de l'in- 
tervenant en francisation. Mais ce dernier doit également se méfier des écueils liés au processus et à la nature des changements à induire.

En effet, la francisation n'a pas pour objet de plier la langue à des usages individuels ou à des usages particuliers à une collectivité, mais bien d'amener cette collectivité à utiliser la langue d'usage au sein de la communauté francophone. Aussi, ce processus présente-t-il des aspects négatifs, du moins dans sa phase initiale. Devoir apprendre à l'Autre que la langue qu'il parle n'est pas celle qu'il croit parler n'a rien d'agréable pour personne. Aussi faut-il procéder avec ménagement. Toutes proportions gardées, l'intervenant en francisation se trouve dans la situation du médecin forcé d'apprendre à un malade qui s'ignore la nécessité d'une intervention chirurgicale. Comme tout médecin qui se respecte se doit d'informer son malade de la nature de son mal et de celle de l'intervention qu'il doit subir, tout intervenant en francisation se doit d'informer tous les intéressés de la nature des changements à apporter.

Chacun sait que le choix de la qualité de la langue à implanter revient en définitive à la direction de l'entreprise. Mais encore faut-il qu'on la munisse de toutes les informations nécessaires à sa décision.

Nous sommes en Amérique du Nord. Nous savons bien qu'une image est attachée à certains titres, fruits d'une traduction littérale. Mais que l'intervenant en francisation se méfie d'un dérapage vers la «franglicisation" dont pourrait être la cause son souci de complaisance à l'endroit de la hiérarchie ou de prestige personnel. Ayant cautionné ou amorcé la «franglicisation», quel moyen aurait-il de la contrer?

Ces écueils contournés, s'amorcera la phase épanouissante de la francisation, celle où les usagers de la langue en découvrent les avantages. Car la langue nous donne les outils voulus pour appréhender le réel et articuler notre pensée. En se sens, elle est porteuse d'efficacité et de rentabilité. On ne le répétera jamais assez!

Julie DesgaGné 